\title{
Mode suppression in a microcavity solid-state dye laser
}

Sergei Popov

Sébastien Ricciardi

Ari T. Friberg

Sergey Sergeyev
Royal Institute of Technology (KTH), Department of Microelectronics and Applied Physics, Electrum 229, SE-164 40 Kista, Sweden

Royal Institute of Technology (KTH), Department of Microelectronics and Applied Physics, Electrum 229, SE-164 40 Kista, Sweden

Royal Institute of Technology (KTH), Department of Microelectronics and Applied Physics, Electrum 229, SE-164 40 Kista, Sweden

Waterford Institute of Technology, Cork Road, Waterford, Ireland

A solid-state dye laser with a microcavity whose size is comparable to the lasing wavelength, is modeled by means of the finite element method. The position of the pumping source affects the lasing mode spectrum. In comparison with a single point source at the edge of the cavity, a random distribution of excitation sources in the central gain part of the microcavity leads to suppression of odd longitudinal modes and to higher output efficiency of the laser radiation in even modes. The modeling and simulation results are explained by simple physical considerations. [DOI: 10.2971/jeos.2007.07023]

Keywords: microcavity, solid-state dye laser, mode depletion

\section{INTRODUCTION}

The rapid development of nano-sciences and bio-applications has stimulated interest in micro- and nano-scaled, highly efficient light emitters. Several tasks in sensing and monitoring techniques, such as drug screening, massive testing of biological specimens, or implementation of 'lab-on-a-chip' systems, require the use of tunable coherent light sources [1]-[4]. Besides different types of semiconductor lasers and LEDs, dye lasers offer a number of the required features: high brightness and coherence, narrow line width of radiation, and broadband wavelength tunability in the visible part of the electromagnetic spectrum [5]. The latter property is an essential feature for applications in bio-sciences, medicine, and chemistry $[6,7]$. Recently, new advances have been reported in the experimental realization of micro-structured tunable dye lasers $[8,10]$. In particular, the operation of micro-fluidic dye lasers with the cavity size in the millimeter regime or slightly smaller was demonstrated. However, the use of a liquid gain medium imposes restrictions as regards further down-sizing of such microcavities, to be implemented in high-density integrated photonic devices.

Suitable candidates to produce really micro- (or nano-)sized, tunable coherent light sources in the visible region are solidstate dye lasers. Recent developments in polymer physics and considerable improvements of dye properties, such as stability against photo-bleaching and clusterization at high concentrations, increase the attractiveness of solid-state dyes as laser media for micro- and nano-photonics applications [11]-[13]. Among additional useful characteristics of such microcavity dye lasers are the ease of operation and inexpensive manufacturing, e.g., by the nano-imprinting lithography technique.
In this paper, we describe the modeling and simulations of solid-state dye lasers with a microcavity of a typical scale required for micro-(nano-)photonics applications, $4-10 \mu \mathrm{m}$. As the gain material, we consider a solid solution of Rh6G dye embedded in a PMMA polymer host matrix. For the numerical calculations, we use the finite element method (FEM) implemented in the Comsol Multiphysics package; it provides accurate solution for the electromagnetic mode structure in a microcavity dye laser of this type.

Considering a random distribution of point excitation sources within the gain section the simulation results indicate significant depletion of the odd longitudinal cavity modes and enhancement of the even laser modes. We explain these phenomena in simple physical terms based on cavity symmetry. The suppression of every other mode in a microscale dye cavity is, to our knowledge, reported here for the first time. The main implications are briefly pointed out. In particular, the phenomenon can improve the tunability of the device, since it broadens the free spectral range (FSR). And it also further enhances a smooth operation of the cavity without mode hopping. Additionally, it allows minimizing the number of mode selective components to provide single-mode operation. All these facts can considerably simplify the design and decrease the manufacturing costs of tunable microcavity lasers. 


\section{MODEL FOR A SOLID-STATE DYE LASER WITH PLANAR TOPOLOGY}

As a starting point for our analysis, we take the model of a liquid dye microcavity ring laser demonstrated recently [14]. Similar lasers with planar topology and typical cavity sizes of several hundreds of micrometers have been realized experimentally [8]. Despite the successful demonstration of laser operation, micro-fluidic dye lasers may have certain potential problems related to further miniaturizing and subsequent implementation in micro- and nano-scaled photonic devices. Reliable control of the liquid gain medium, for instance, to establish the operation and/or real-time tunability of the laser, can work well in sub-millimeter scales [8]. However, it might be difficult to realize such devices with significantly smaller cavity sizes owing to the changes in specific liquid properties, e.g., viscosity, surface tension, and thermo-mechanical coefficients.

Hence, we have opted to investigate the applicability of this model for solid-state dye lasers with the cavity size comparable to the operation wavelength. We substitute the microfluidic channel (containing a liquid dye solution and placed between the triangle-shaped parts of the coupled microcavity, see Figure 1) with a solid polymer bar embedded with dye molecules. Low concentration solid dye solution is rep-

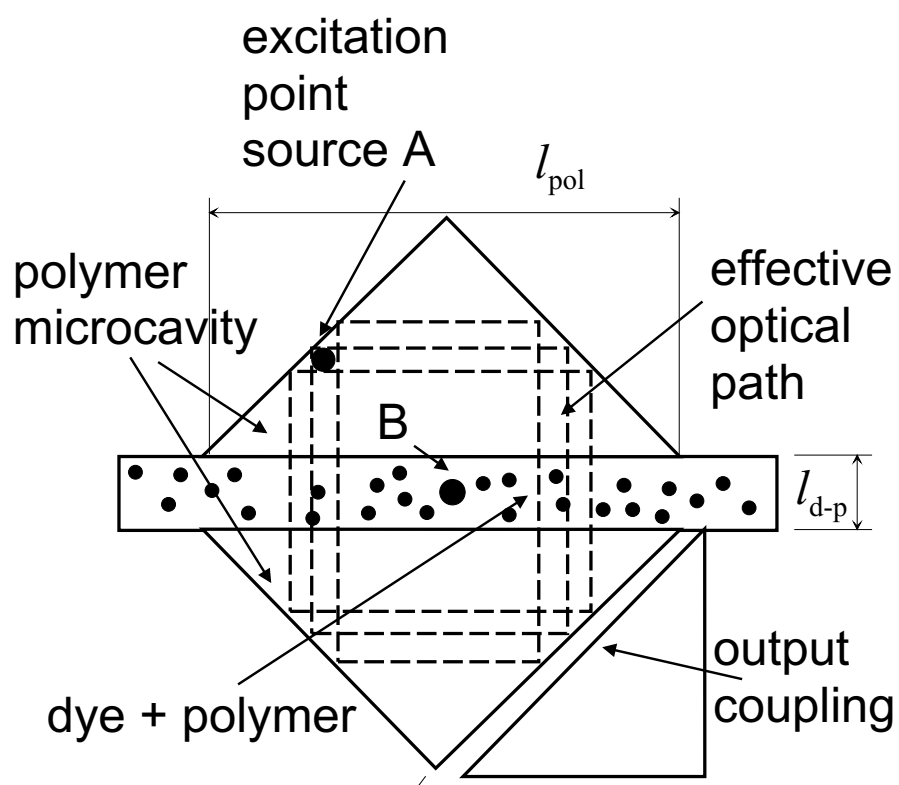

$$
l_{\text {air }}
$$

FIG. 1 Layout of the microcavity solid-state dye laser, illustrating the optical paths of the effective cavity modes (dashed lines) and the polymeric gain material that contains dye molecules (random dots in the central slab). Dots A and B represent particular single-source configurations.

resented with a large number of dye molecules as point excitation sources randomly allocated inside the polymer bar, instead of a single-point excitation (SPE) close to the edge of the cavity as in Ref. [14] (point A in Figure 1). This approach with a multi-point excitation (MPE) source corresponds more closely to a real physical arrangement, in which the dye laser with a planar topology is optically pumped through the top facet [8].
The microlaser consists of several components that are placed in contact with each other, as illustrated in Figure 1: two triangular main half-cavity parts, the central gain slab, and an output coupling prism. We assign typical values for the refractive indices of the various parts of the device, i.e., $n_{d-p}=1.43$ for the dye-polymer gain section and $n_{p o l}=1.6$ for the polymer (SU-8) triangles of the microcavity at the wavelength of $500 \mathrm{~nm}$. To account for the dispersion properties of the materials within the bandwidth of interest $(455-600 \mathrm{~nm})$, we make use of an accurate approximation of the refractive index based on the Sellmeier coefficients [15]. Optical losses for the materials in our cavity model are constant in the visible range, and have values of $0.65 \mathrm{~dB} / \mathrm{cm}$ for PMMA and $1.4 \mathrm{~dB} / \mathrm{cm}$ for SU-8 [16]. To investigate the cavity mode patterns rather than dynamical operation of the laser, and to keep the model simple, we ignore the amplification in the gain material.

The dye laser in the reference model had a cavity of a typical size of hundreds of $\mu \mathrm{m}$ and it was numerically simulated for the microwave spectral band; one assumed that it could simply be scaled down to the optical regime [14]. In view of this, in our analysis we reduce the microcavity size down to $4 \mu \mathrm{m}$ while keeping a similar wavelength / cavity dimension ratio. We use the FEM solver implemented in Comsol Multiphysics to find steady-state solutions for the electric field $\mathbf{E}$ at different microcavity mode frequencies $\omega$. We employ a 2D model for transverse-electric (TE) waves, where the electric field vector only has a component perpendicular to the plane cavity. This allows a simplified model and ensures the necessary computing power for finding the solution of the Helmholtz equation

$$
\nabla^{2} \mathbf{E}+\mu_{r} \epsilon_{r}(\omega / c)^{2} \mathbf{E}=0,
$$

where $\mu_{r}$ is relative permeability (equal to 1 for our materials), $\epsilon_{r}$ is relative permittivity, and $c$ is the vacuum speed of light. The conductivity of the materials is zero. The boundary conditions for all the interfaces in the cavity also require the continuity of the tangential component of the magnetic field $\mathbf{H}=\left(i \omega \mu_{0} \mu_{r}\right)^{-1} \nabla \times \mathbf{E}$, i.e.,

$$
\mathbf{n} \times \mathbf{H}=0,
$$

where $\mathbf{n}$ is the unit vector normal to the boundary interfaces.

The finite size of the modeling area necessitates one more boundary condition (at the borders limiting the simulation area, not shown in Figure 1), which corresponds to a damped propagation of the electromagnetic field out of the microcavity. This condition ensures that no electric field reflects back onto the microcavity from the working area boundaries. It consists of two parts: one accounts for the continuity of the electric and magnetic fields at the border, whereas another one simulates rapid attenuation of the field behind the border, ensuring less than $10^{-6}$ back reflectance to the simulation area. These properties are realized in the perfect matching layer (PML) feature of Comsol Multiphysics.

As regards excitation, optical pumping of the dye molecules in the dye-polymer section generates radiating dipoles with different spatial orientations. In the 2D topology of the microcavity, these dipoles act as point radiation sources that create harmonic TE waves of frequency $\omega$ (not necessarily resonance 
frequency) [17]. In a homogeneous region the fields can be represented as $\mathbf{E}_{0}\left(\mathbf{r}-\mathbf{r}_{n}\right)$, where $\mathbf{r}_{n}(n=1, \ldots, N)$ are a random spatial distribution of $N$ point sources within the dyepolymer gain section. The form of the electric field $\mathbf{E}_{0}\left(\mathbf{r}-\mathbf{r}_{n}\right)$ is the same for all radiators, and for plane-wave pumping they can all be assumed to be in phase. In Comsol Multiphysics the point sources are specified by giving the field amplitudes $\mathbf{E}_{0}\left(\mathbf{r}_{n}\right)$ at the locations (mesh points) of the sources.

To model the full spectrum and to identify the microcavity resonance modes, we need to solve the problem for all frequencies within the region of the dye luminescence, between 500 and $660 \mathrm{THz}(455-600 \mathrm{~nm})$. All media are taken as homogeneous, and the solution accounts fully for the radiation, diffraction, and scattering (at all boundaries) properties of the TE electromagnetic field. A script including a parametrical solver is used to find the resonance frequencies and mode patterns for the microcavity dye laser. An optimized mesh of about 30000 Lagrange quadratic elements for each frequency is used to ensure the necessary accuracy and computer resources. For the demonstration and discussion of the results, it is more convenient to represent the microcavity spectra as a function of mode numbers rather than frequencies.

Since the microcavity dimensions are roughly an order of magnitude larger than the wavelengths of interest, we use a ray tracing approach to find an averaged effective length of the mode optical paths

$$
L_{e f f}=2\left(n_{d-p} l_{d-p}+n_{p o l} l_{p o l}\right),
$$

where $n_{d-p}$ and $n_{p o l}$ are the refractive indices of the dyepolymer gain slab and polymer half-cavities, whereas $l_{d-p}$ and $l_{p o l}$ are the thicknesses of the dye-polymer slab and the length of the polymer triangle side adjacent to the dyepolymer slab, respectively (see Figure 1). The output coupling of the radiating modes occurs through an air gap $l_{\text {air }}$ at the boundary $\Gamma$ by means of catching the evanescent field outside the cavity. Using the value $l_{p o l}=4 \mu \mathrm{m}$, whereas $l_{d-p}$ is kept at about $20 \%$ of $l_{p o l}$ and $l_{\text {air }}$ at $3 \%$ of $l_{p o l}$, we obtain effective optical paths for the modes as long as $L_{e f f} \sim 15 \mu \mathrm{m}$. To calculate the mode spacing, mode numbers, and resonance frequencies, relations similar to those in Ref. [14] are applied. Using the phase matching condition, the mode numbers are found from following expression

$$
m=\frac{1}{2 \pi}\left(v_{m} L_{e f f} \frac{2 \pi}{c}+\phi\right),
$$

where we consider $m$ rounded to the nearest positive integer (mode number), $v_{m}$ is the (linear) resonance frequency, $L_{e f f}$ is the effective optical path from Eq. (3), and $\phi$ is the phase change due to the reflections at the microcavity walls (which follows from the Fresnel coefficients for the complex amplitude of the electric field) $[14,17]$.

\section{RESULTS: DEPLETION OF ODD AND ENHANCED EFFICIENCY OF EVEN RESONANCE MODES}

To find the mode spectra for the microcavity lasers of the two source configurations (SPE and MPE pumping schemes), we run the Comsol solver in parametric mode, using the cavity mode frequency as a variable parameter. When the microcavity dye laser is modeled with the multi-point excitation source (Figure 1), we obtain noticeable physical features not observed in the original single-point excitation model: odd longitudinal modes are significantly depleted, while the even modes are strongly enhanced. The main result of the simulations is shown in Figure 2, where we compare the resonance spectra of the longitudinal modes for two models: one is for our modified MPE design and the other one is for the SPE scheme considered in Ref. [14].

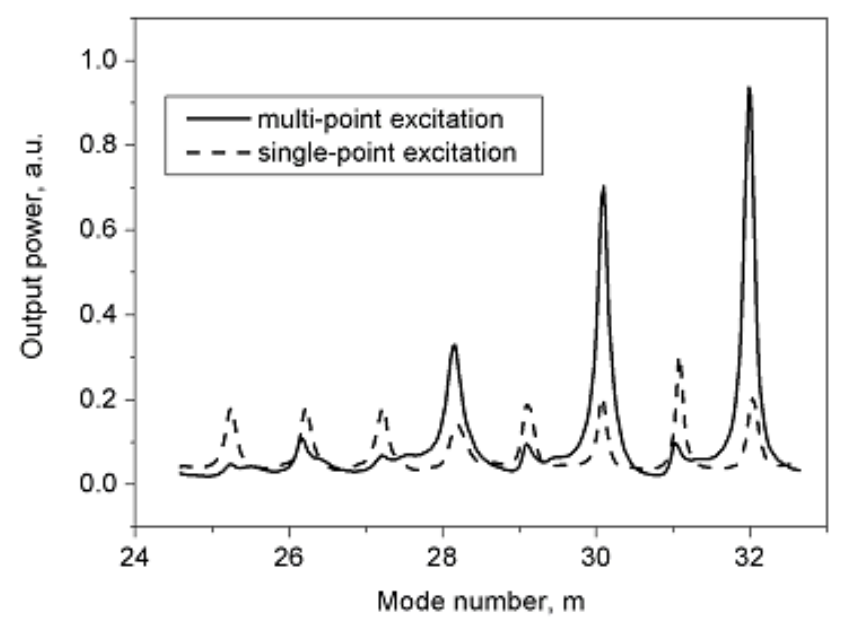

FIG. 2 Longitudinal modes in the microcavity solid-state laser for different excitation schemes: single-point at the edge of the cavity (solid curve) and multi-point inside the central gain slab (dashed curve). The output power is normalized by the number of excitation points.

The graphs display the total power (in arbitrary units) of the electric field integrated along the out-coupling boundary $\Gamma$ (Figure 1). For a proper comparison, and especially to evaluate the output coupling efficiency, the results are normalized, i.e., the values for the MPE arrangement are divided by $N$, the number of SPE sources. We choose $N=500$ as a reasonable number which simulates a rather homogeneous distribution of dye molecules in the gain section, and simultaneously ensures sufficient computer resources to run the calculations over the more than $160 \mathrm{~nm}$ wide spectral bandwidth. Thus, the total pump power is the same as for the SPE design and it is evenly distributed among 500 point sources randomly allocated in the polymer-dye slab.

Strictly speaking, the longitudinal mode numbers for the 2D micro-sized cavity are not to be interpreted as directly as the numbers of nodes and antinodes of one standing wave, like in the case of $1 \mathrm{D}$ long laser cavity. However, here it is possible and instructive to consider that the mode numbers refer to the effective optical path length in the central region of the cavity (as explained in Section 2), since the parity of these numbers can help us to explain the relation between the mode properties shown in Figure 2, the microcavity symmetry, and the allocation of the excitation sources.

No matter where an excitation source is located inside the cavity, the existence of the resonance modes requires satisfying 
the phase-matching conditions for wave round trips along the closed optical path. The microcavity with the gain section inserted between the two parts (not considering the output coupling prism) possesses spatial symmetry about the horizontal axis through the central slab. The position of the excitation source relative to this axis and the mode parity (treated as the parity of the mode number) play an important role on how efficiently the pump energy is coupled into the cavity modes.

To clarify this relationship, we consider the mode spectra for two different positions of the SPE sources placed far from and close to the horizontal symmetry axis, i.e., points $\mathrm{A}$ and $\mathrm{B}$ in Figure 1, respectively. The corresponding mode spectra are illustrated in Figure 3. The graphs show that for point B

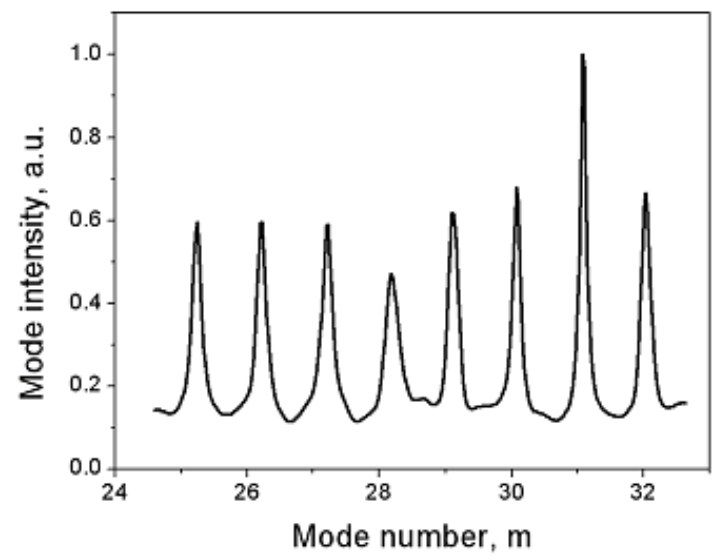

(a)

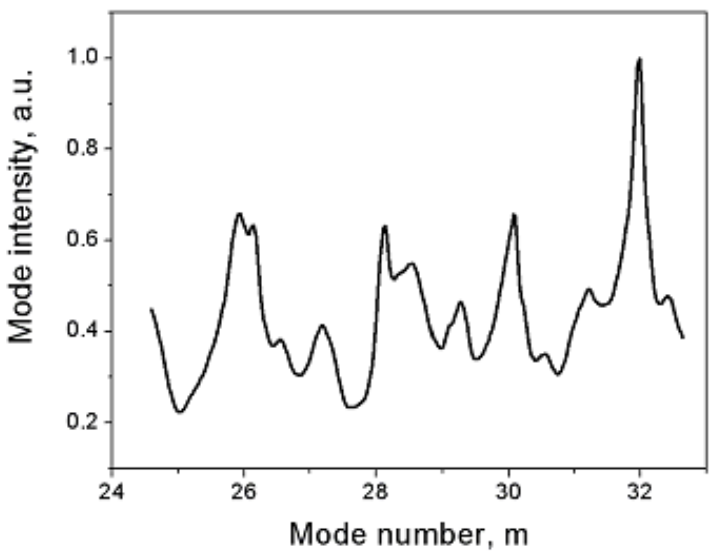

(b)

FIG. 3 Microcavity mode spectra for SPE sources allocated (a) at the edge (point A in Figure 1) and (b) in the center (point B in Figure 1) of the microcavity.

(in the central part of the cavity) the intensities of the modes with odd mode number are considerably suppressed [Figure $3 b]$, whereas the spectrum for point A (located close to the edge) does not exhibit any such dependence on the mode number parity [Figure 3a]. We can reasonably explain this phenomenon with different coupling efficiencies of the SPE pumping wave into the cavity modes with odd and even numbers using the mutual symmetry between the cavity modes and the location of the excitation source.

First we note that when the propagation distance is sufficiently large, the fields $\mathbf{E}_{0}\left(\mathbf{r}-\mathbf{r}_{0}\right)$ created by point sources behave in phase as 2D spherical waves [17]. When the excitation source, point $\mathrm{A}$ in Figure $4 \mathrm{a}$, is placed close to the cavity edge, the phase fronts of the spherical wave (solid line) cross the mode path (dashed line) at points which in all likelihood have different phases for the even and the odd numbered modes, for example, pairs $1-2$ and $3-4$. Although, by symmetry, the even numbered modes would have the same phase at point pairs $1-1^{\prime}$ and $3-3^{\prime}$, the phase of the excitation wave would not match. In such a case, therefore, no favorable conditions are created for the coupling of the pump excitation into the modes with different parities. The result then is a fairly even distribution of the output intensity among the odd and even modes [Figure 3a].
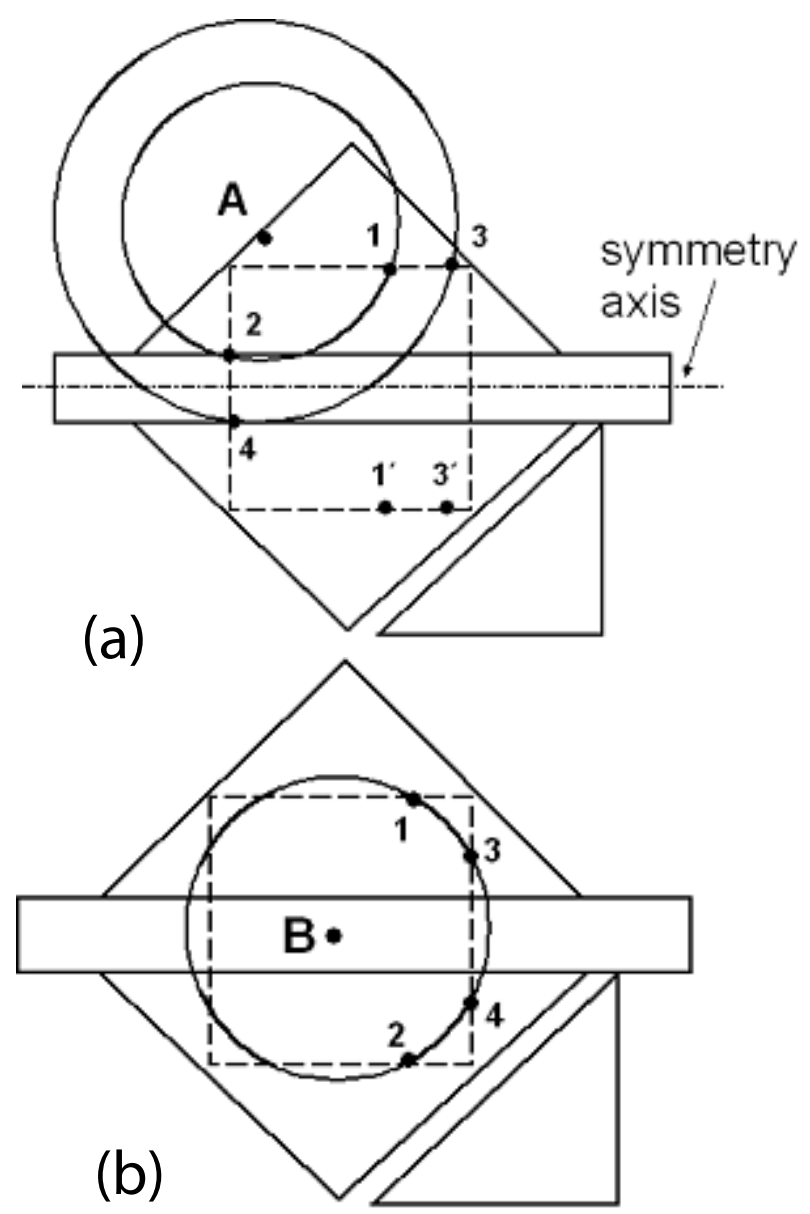

FIC. 4 Schematic representations of the overlapping between the excitation spherical wave and the microcavity modes: (a) the source is placed at the cavity edge (point A in Figure 1); (b) the source is placed in the cavity center (point B in Figure 1).

Next, we consider the excitation source, point B in Figure 4b, at the center of the cavity. For the even numbered resonance modes, the symmetrical cavity geometry leads to the same phase of the optical wave (dashed line) at every pair of points placed symmetrically at equal distances above and below the horizontal axis, for example, point pairs $1-2$ and $3-4$. Since the SPE source emits a spherical wave (solid line), such a circumstance ensures an efficient transfer of the excitation energy into the cavity mode at these points due to constructive superposition. For the same working conditions, the odd numbered modes do not provide similar phase matching between the cavity modes and the spherical excitation waves, since the phases of the mode wave at point pairs $1-2$ and $3-4$ are no longer equal [18]. Thus, the efficiency of the exci- 
tation coupling into the odd numbered modes is weaker [Figure $3 b]$.

With multiple source-point excitation in the dye-polymer bar, the electric field patterns demonstrate the relation between the different mode number parity and the symmetry of the modes within the microcavity, as illustrated in Figure 5. Comparing the electric field patterns for the modes $m=32$ [Figure 5a] and $m=31$ [Figure 5b], one can easily see that the odd mode pattern is slightly skewed in the central part of the cavity and lacks the symmetry of the wave nodes and antinodes in the upper and lower halves of the cavity. The large number of the points constituting the MPE source in the central section of the microcavity amplifies (and averages) the SPE effects. The fields in the out-coupling prism regions clearly demonstrate that modes propagating in both (opposite) directions are included. Also the difference of the pump excitation coupling into the modes with different parities is evident in the different strengths of the even and odd modes exactly at the edge of the out-coupling prism.

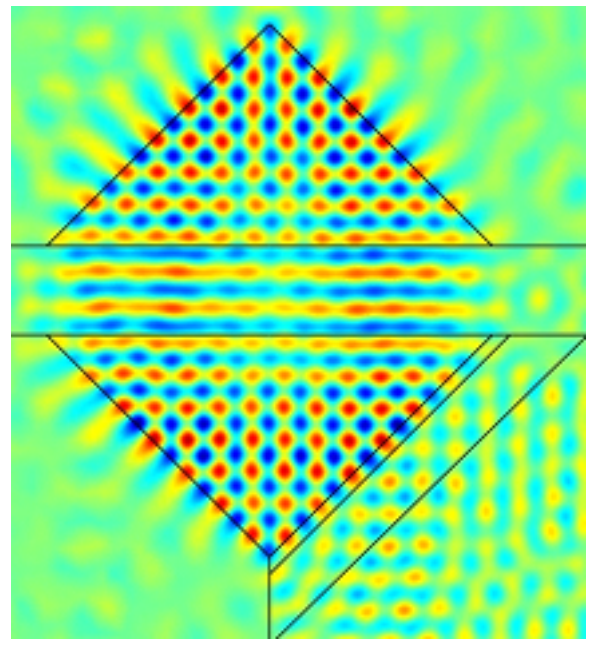

(a)

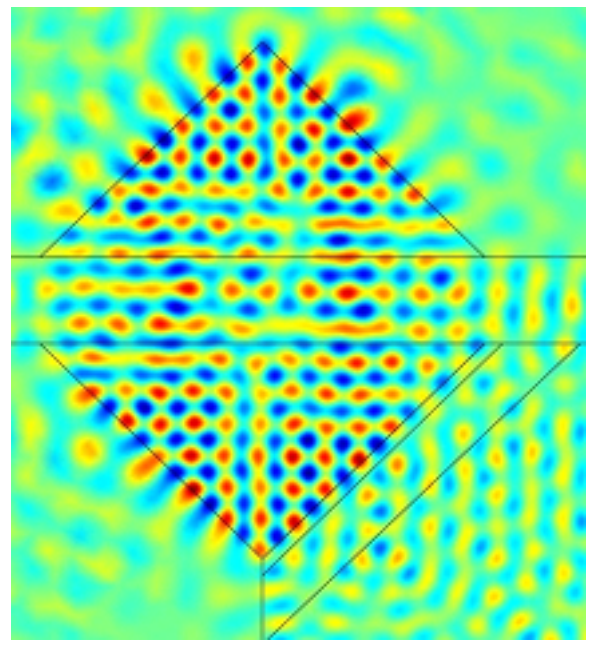

(b)

FIG. 5 Electric field patterns for the even, $m=32$ (a) and odd, $m=31$ (b) microcavity modes excited by MPE source consisting of 500 elementary sources in the dye-polymer bar (see Figure 1). Maximum, minimum, and zero values of the electric field amplitudes are encoded with red, blue, and green colors respectively.
If the excitation source in the SPE scheme is placed outside the central slab, the electric field patterns clearly manifest the absence of the symmetry for both modes (see Figure 6) and no preferable conditions exists for the excitation coupling into the even modes.

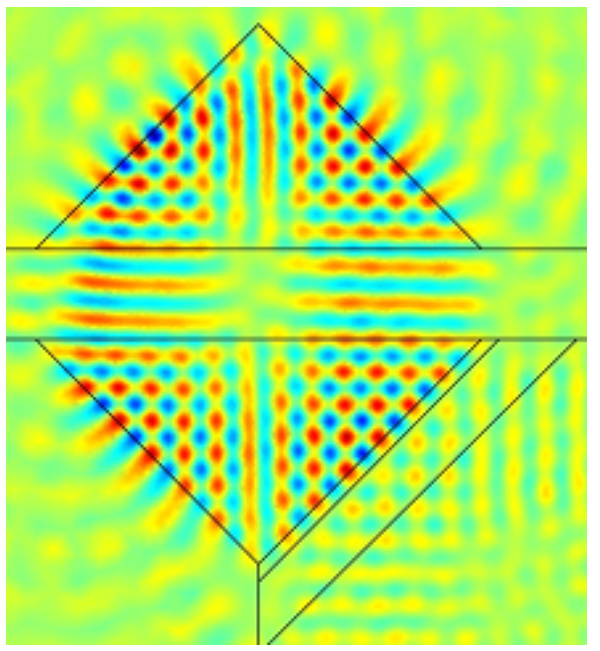

(a)

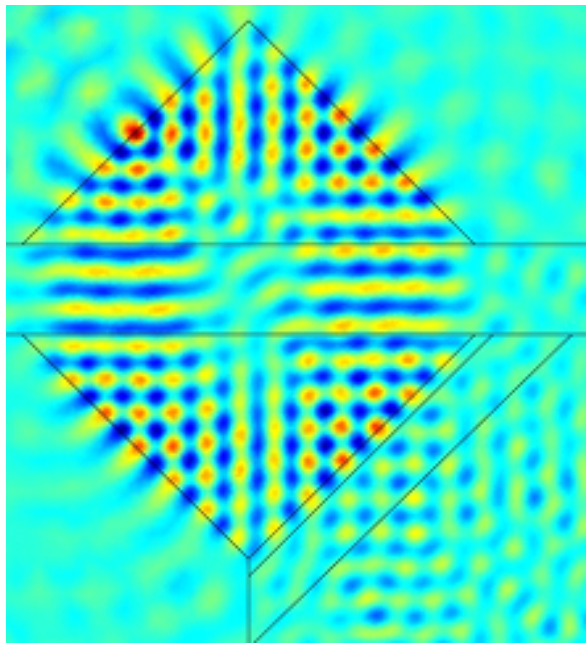

(b)

FIG. 6 Electric filed patterns for the even, $m=32$ (a) and odd, $m=31$ (b) microcavity modes excited by SPE source placed at the cavity edge (point $A$ in Figure 1). The color scheme is as in Figure 5.

An appropriate allocation of the gain (excitation) part of the solid-state dye microlaser can be used, for example, to adjust the mode spectrum according to particular application requirements. As we have demonstrated, the central symmetrical position of the gain section can suppress every other mode and thus increase the free spectral range and the tunability of such lasers without mode hopping.

\section{CONCLUSIONS}

In this paper, we have considered modeling a solid-state dye laser with the cavity size slightly larger than the radiation wavelength. The design, when the gain material is simulated 
as a multi-point excitation source placed between the two similar parts of the cavity, demonstrate the suppression of odd modes and the increased output radiation in even laser modes. We attribute this effect to the symmetry relation between the cavity shape and allocation of the excitation sources (the gain section) within the cavity. The mode suppression effect can be used to tailor the tunability of microcavity lasers, since it increases the free spectral range while keeping the same cavity size. The model and numerical calculations of the resonance mode patterns are rigorous and thus apply, in principle, even to very small cavities. In our analyses the effective round-trip optical path length is about $15 \mu \mathrm{m}$, considerably shorter than in previous related investigations.

When the cavity sizes are comparable with the wavelength of the radiation, and the (geometrical optics) operation mode numbers are low as a consequence, the mode frequencies are sensitive to small variations of the microlaser geometry. A controllable change of the cavity size and geometry can be used to realize wavelength tuning of the radiation.

Since many polymers possess low absorption and dispersion at visible wavelengths, there exists a choice of materials suitable for hosting dyes to create the laser gain medium. On the other hand, to exploit such materials with advantageous nano-imprinting lithography techniques, other mechanical and chemical properties (such as compatibility with stamp and mask materials and sticking features) should be taken into account in the search of optimized compositions.

Besides the factors based on geometry, a particular composition and distribution of the polymer-dye gain material can influence the mode depletion behavior. This effect requires more accurate simulations, including modeling inhomogenous radiation patterns for dye molecules and amplification of the optical wave.

\section{ACKNOWLEDGEMENTS}

The authors affiliated with Royal Institute of Technology (KTH) acknowledge financial support from the Swedish Foundation for Strategic Research (SSF).

\section{References}

[1] I. M. White, Z. Hongying, J. D. Suter, N. M. Hanumegowda, H. Oveys, M. Zourob, and F. Xudong, "Refractometric sensors for lab-on-achip based on optical ring resonators" IEEE Sens. J. 7, 28-35 (2007).
[2] H. Craighead, "Future lab-on-a-chip technologies for interrogating individual molecules" Nature 442, 387-393 (2006).

[3] D. Psaltis, S. R. Quake, and C. Yang, "Developing optofluidic technology through the fusion of microfluidics and optics" Nature 442 , 381-386 (2006).

[4] M. P. MacDonald, G. C. Spalding, and K. Dholakia, "Microfluidic sorting in an optical lattice" Nature, 426, 421-424 (2003).

[5] F. J. Duarte, ed., Tunable Lasers Handbook (Elsevier, Amsterdam, 1995).

[6] B. N. G. Giepmans, S. R. Adams, M. H. Ellisman, and R. Y. Tsien, "The fluorescent toolbox for assessing protein location and function" Science 312, 217-224 (2006).

[7] P. N. Prasad, Introduction to Biophotonics (Wiley, New York, 2003).

[8] S. Balslev and A. Kristensen, "Microfluidic single-mode laser using high-order Bragg grating and antiguiding segments" Opt. Express 13, 344-351 (2005).

[9] B. Bilenberg, T. Rasmussen, S. Balslev, and A. Kristensen, “Realtime tunability of chip-based light source enabled by microfluidic mixing" J. Appl. Phys. 99, 23102:1-5 (2006).

[10] Y. Cheng, K. Sugioka, and K. Midorikawa, "Microfluidic laser embedded in glass by three-dimensional femtosecond laser microprocessing" Opt Lett. 29, 2007-2009 (2004).

[11] A. Costela, I. Garcia-Moreno, D. del Agua, 0. Garcia, and R. Sastre, "Silicon-containing organic matrices as hosts for highly photostable solid-state dye lasers" Appl. Phys. Lett. 85, 2160-2162 (2004).

[12] F. Duarte and R. 0. James, "Tunable solid-state lasers incorporating dye-doped, polymer-nanoparticle gain media" Opt. Lett. 28, 20882090 (2003).

[13] A. Costela, I. Garcia-Moreno, C. Gomez, 0. Garcia, and R. Sastre, "New organic-inorganic hybrid matrices doped with rhodamine $6 \mathrm{C}$ as solid-state dye lasers" Appl. Phys. B 75, 827-833 (2002).

[14] M. Hansen-Gersborg, S. Balslev, and N.A. Mortensen, "Finiteelement simulation of cavity modes in a microfluidic dye ring laser" J. Opt. A 8, 17-20 (2006).

[15] H. El Rhaleb, N. Cella, J. P. Roger, D. Fournier, A. C. Boccara and A. Zuber, "Beam size and collimation effects in spectroscopic ellipsometry of transparent films with optical thickness inhomogeneity" Thin Solid Films 288, 125-131 (1996).

[16] http://www.microchem.com/products/su_eight.htm.

[17] E. Hecht, Optics, 4th ed. (Addison Wesley, San Francisco, 2002).

[18] We emphasize that while for cavity modes that contain an even number of nodes the wave (by symmetry reasons) is symmetric about the horizontal axis, the waves with an odd number of nodes are not anti-symmetric about this line, but rather more complicated phase relations may arise. 